\title{
Elaboración de un instrumento para medir el capital intelectual en una institución de educación media superior, en el estado de Tabasco
}

\section{Development of an instrument to measure intellectual capital in an institution of higher secondary education, in the state of Tabasco}

GARCÍA-REYES, David Antonio †*, PÉREZ-PÉREZ, Iris Cristel, ELISEO-DANTÉS, Hortensia y MENDOZA-WILSON, Ángela del Carmen

Tecnológico Nacional de México, Av. Universidad 1200, Col. Xoco, Ciudad de México, Alcaldía Benito Juárez C.P. 03330. Instituto Tecnológico de Villahermosa. Km. 3.5 Carretera, Villahermosa - Frontera, Cd Industrial, 86010 Villahermosa, Tab.

ID $1^{\text {er }}$ Autor: David Antonio, García-Reyes / ORC ID: 0000-0002-6083-079X, Researcher ID Thomson: D-4836-2018, CVU CONACYT ID: 883868

ID $1^{\text {er }}$ Coautor: Iris Cristel, Pérez-Pérez / ORC ID: 0000-0003-3120-5597, Researcher ID Thomson: G-1891-2018, CVU CONACYT ID: 843577

ID $2^{\text {do }}$ Coautor: Hortensia, Eliseo-Dantés / ORC ID: 0000-0003-4006-4669, Researcher ID Thomson: F-6749-2018, CVU CONACYT ID: 411079

ID $3^{\text {er }}$ Coautor: Ángela del Carmen, Mendoza-Wilson / ORC ID: 0000-003-4228-725X, PubMed ID: 42f5b1bfdf4feb.17da25e4bb05217346c09, CVU CONACYT ID: 914348

\section{Resumen}

El estudio del capital intelectual es de suma importancia para las organizaciones actuales. Por lo tanto, en el presente trabajo de investigación se llevará a cabo el estudio integral en el área académica de una institución de educación media superior, en el estado de Tabasco, para el desarrollo de un instrumento de medición. Se propone diseñar un instrumento, el cual se considera un híbrido al estar formado por información intangible que es interpretada por el investigador y traducida a través de una escala de Likert a un dato cuantitativo que permite ubicar el factor de estudio de una manera clara para determinar su grado de influencia en la generación de capital intelectual. Además dichos factores son tomados de expertos del contexto de estudio cuya experiencia les permite opinar. Dicha información es incluida en una tabla de doble entrada que permite encontrar los factores claves de incidencia en el tema de estudio. Una vez identificados los factores, se analizarán y se realizará una propuesta de desarrollo que permita el mejoramiento integral de la institución de educación media superior en el área académica, para así, contribuir al Capital Intelectual.

Capital intelectual, Instrumento, Medición

\begin{abstract}
The study of intellectual capital is of the utmost importance for current organizations. Therefore, in the present research work the integral study will be carried out in the academic area of an institution of higher secondary education, in the state of Tabasco, for the development of a measuring instrument. It is proposed to design an instrument, which is considered a hybrid as it is formed by intangible information that is interpreted by the researcher and translated through a Likert scale to a quantitative data that allows to locate the study factor in a clear way to determine its degree of influence in the generation of intellectual capital. In addition, these factors are taken from experts in the study context whose experience allows them to give their opinion. This information is included in a double entry table that allows to find the key factors of incidence in the subject of study. Once the factors have been identified, a development proposal that allows the integral improvement of the institution of higher secondary education in the academic area will be analyzed and made, in order to contribute to Intellectual Capital.
\end{abstract}

Intellectual capital, Instrument, Measurem

Citación: GARCÍA-REYES, David Antonio, PÉREZ-PÉREZ, Iris Cristel, ELISEO-DANTÉS, Hortensia y MENDOZAWILSON, Ángela del Carmen. Elaboración de un instrumento para medir el capital intelectual en una institución de educación media superior, en el estado de Tabasco. Revista Teoría Educativa. 2019. 3-7: 34-37.

\footnotetext{
* Correspondencia del Autor (Correo electrónico: garcia.reyees@gmail.com)

$\dagger$ Investigador contribuyendo como primer autor.
} 


\section{Introducción}

La información y el conocimiento es una de las armas más valiosas y competitivas de nuestra época. Es atrevido decir que el conocimiento es aún más valioso que una gran cuenta en el banco o una gran fábrica, ya que las empresas que triunfan en el actual mundo globalizado, son aquellas que poseen la mejor información y saben emplearla de una manera eficiente.

Actualmente, dicha afirmación se puede ejemplificar con diversas empresas transnacionales que tienen un éxito rotundo las cuales, lograron su grandeza, no por ser más ricas, sino por poseer algo mucho más valioso, Capital Intelectual.

El concepto de Capital Intelectual ha sido utilizado en la literatura académica desde hace muchos años; es un tópico sobre el cual, el interés de las organizaciones ha crecido rápidamente en los últimos años. En este sentido, Bontis (1998) afirma que "el capital intelectual ha sido considerado por muchos, definido por algunos, entendido por pocos y formalmente valorado por prácticamente nadie", lo cual representa uno de los desafíos más importantes para las empresas actuales.

Bradley (1998), argumenta que el capital intelectual consiste en la capacidad para transformar el conocimiento y los activos intangibles en recursos que crean riqueza tanto en las empresas como en los países. De igual modo, para Edvinsson y Sullivan (1996) y Sullivan $(1999,2001)$ el capital intelectual es aquel conocimiento que puede ser convertido en beneficio en el futuro y que se encuentra formado por recursos tales como las ideas, los inventos, las tecnologías, los programas informáticos, los diseños y los procesos. Tomando como base esta definiciones, se puede concluir que el Capital Intelectual es la suma de conocimientos que poseen todos los colaboradores de una organización y le dan a ésta, una ventaja competitiva.

Para el presente trabajo de investigación se indaga de manera integral en el área académica de una institución de educación media superior, en el estado de Tabasco, y así, ubicar los factores que determinan una influencia determinante, en la generación de capital intelectual, para concluir con el desarrollo de una propuesta de mejora.

\section{Planteamiento del problema}

Las instituciones de educación media superior, al igual que todas las instituciones que se centran en la educación, en el estado de Tabasco enfrentan varios cambios y retos académicos de suma importancia que están siempre enfocados a la mejora continua para brindar una educación de alta calidad a los jóvenes de la región y todos los actores que intervienen en el proceso.

Es por ello, una de las razones por las cuales se desea valorar la incidencia que tiene el capital intelectual dentro de la entidad, porque este es uno de sus activos intangibles más valiosos siendo este un factor fundamental para enfrentar dichos cambios y retos institucionales. Por esta razón es importante implementar una metodología que nos permita valorar el capital intelectual. Es importante que la institución este consciente de que su capital intelectual esté presente en todas las dependencias, y que necesaria y frecuentemente este, está impactando directamente a docentes y estudiantes de los diferentes programas académicos.

En los últimos años, la importancia del capital intelectual dentro de una organización ha ido tomando cada vez más fuerza, y lo que este aporta y representa para la eficiencia de la misma. No obstante hay que tener claridad que las instituciones tanto públicas como privadas, y sociedad en general carecen totalmente de sistemas de medición que cada vez se hacen más necesarias para estas nuevas fuentes de riqueza, como lo es el objeto de estudio. Por tal motivo, el valorar el capital intelectual y su identificación llegó a ser objeto de estudio para ser medido cuantitativamente de alguna forma.

Lo que se busca, es que la organización pueda administrar con efectividad sus activos inmateriales presentes en cada dependencia de la organización, caso particular los docentes para que estos puedan ser unos proveedores de conocimiento distintivo, aportando a los estudiantes de la institución y fomentar por medio de la enseñanza, la capacidad de generar investigación, desarrollo y crecimiento competitivo. 


\section{Justificación}

La tendencia de las empresas exitosas que evolucionan en contextos globalizados está cimentada bajo el concepto de activos intangibles, es decir; procesos de creatividad e innovación permanentes, personal con habilidades y destrezas.

Por tanto, es necesario que desde la educación media superior, se empleen las tendencias y los nuevos requerimientos del sector empresarial, ya que es de vital importancia los vínculos que se establezcan con el sector productivo, como motor de desarrollo y dinamización de la economía.

Con el Estudio del capital intelectual en el área académica de una institución de educación media superior, en el estado de Tabasco, se pretende incursionar en un campo emergente a nivel institucional como lo es el Capital Intelectual, realizando diagnósticos internos que permitan determinar cuál es el estado actual de la institución, frente a los procesos de conocimiento y como son estos objetados a nivel externo, para así poder determinar planes y estrategias de mejoramiento, con fines de que la institución responda a las exigencias de un mercado dinámico repercutiendo en la competitividad de las mismas frente a los procesos de internacionalización.

\section{Objetivo General}

Generar una propuesta de desarrollo para la generación de Capital Intelectual, en una Institución de Educación Media Superior, en el estado de Tabasco.

\section{Objetivos Específicos}

1.- Realizar un diagnóstico sobre el capital intelectual, en una Institución de Educación Media Superior, en el estado de Tabasco.

2.- Generar un instrumento que permita identificar los factores determinantes y su grado de influencia en la generación de capital intelectual, en el área académica de una institución de educación media superior, del estado de Tabasco.
3.- Diseñar una propuesta de desarrollo para la generación de Capital Intelectual, en una Institución de Educación Media Superior, en el estado de Tabasco.

\section{Delimitación del problema}

Está contemplado realizarse en el periodo de Agosto de 2019 a Marzo de 2020.

\section{Metas de investigación}

1.- Un instrumento que permita identificar los factores determinantes y su grado de influencia en la generación de capital intelectual, en el área académica de una institución de educación media superior, del estado de Tabasco, para el segundo semestre del año 2019.

2.- Una propuesta de desarrollo para la generación de Capital Intelectual, en una Institución de Educación Media Superior, en el estado de Tabasco, para el primer semestre del año 2020.

\section{Identificación de las variables y formulación de hipótesis}

Las seis variables del contexto inciden en la generación de capital intelectual en el área académica de una institución de educación media superior, del estado de Tabasco; las cuales son:

1) Económica.

2) Social.

3) Cultural.

4) Política.

5) Tecnológica.

6) Ambiental.

\section{Formulación de Hipótesis}

Las variables: económica, social, cultural, política, tecnológica y ambiental inciden en la generación de capital intelectual, en el área académica de una institución de educación media superior, del estado de Tabasco. 


\section{Tipo de investigación}

1.- Documental: Se caracteriza por la selección y recopilación de información por medio de la lectura y crítica de documentos y materiales bibliográficos, de bibliotecas, hemerotecas, centros de documentación e información. Guarda estrecha relación con la investigación histórica, porque se recurre al análisis de documentos como: libros, revistas, prensa, censos, estadísticas, anuarios, películas, diapositivas, planos, discos, fotografías, cintas o grabaciones.

2.- Correlacional: Dado que en la investigación se especifican una serie de variables que inciden en el tema de análisis, las mismas se deben de interrelacionar para poder explorar de manera integral su impacto.

\section{Referencias}

Alonso, G., (2013). Estudio de casos de Capital Intelectual en Empresas Gallegas. Facultade de Ciencias Empresariais e Turismo. Universidade De Vigo.

Bontis, N. (1998). Capital Intelectual: Un estudio exploratorio que desarrolla medidas y modelos. Management Decision. pp. 66-73.

Edvinsson, L. y Sullivan, P. (1996). "Desarrollar un modelo para Gestionar el Capital Intelectual", European Management Journal, Vol. 14, No. 4, pp. 356-364.

Heredia, J., (2019). Influencia del Capital Intelectual en la Competitividad de los Hoteles. Conciencia Tecnológica, núm. 37. Instituto Tecnológico de Aguascalientes, pp. 20-25.

Monagas, M. (2012). El Capital intelectual y la gestión del conocimiento. Ingeniería industrial, 142-150.

Villegas, E., Hernández, M. \& Salazar, B. (2015). La medición del capital intelectual y su impacto en el rendimiento financiero en empresas del sector industrial en México. Universidad Nacional Autónoma de México, Facultad de Contaduría y Administración. (2016).
Sánchez Medina, A. J., Melián González, A. \& Hormiga Pérez, E. (2007). El Concepto del Capital Intelectual y sus dimensiones. Investigaciones Europeas de Dirección y Economía de la Empresa, vol. 13, núm. 2. Academia Europea de Dirección y Economía de la Empresa. pp. 97-111.

Sullivan, P.H. (1999): “Aprovechar el Capital Intelectual", Journal of Knowledge Management, Vol. 3, No. 2, pp. 132-142.

Sullivan, P.H. (2001): Introducción a la gestión del Capital Intelectual, en Sullivan, P.H. (eds.), Rentabilizar el capital intelectual. Técnicas para optimizar el valor de la organización, Paidós empresa, Barcelona 